\title{
Ultrafast All-Optical Modulation in Silicon Optical Fibers
}

\author{
P. Mehta ${ }^{1}$, N. Healy ${ }^{1}$, J.R. Sparks ${ }^{2}$, T.D. Day ${ }^{2}$, P.J.A. Sazio ${ }^{1}$, J.V. Badding ${ }^{2}$, and \\ A.C. Peacock ${ }^{1}$ \\ ${ }^{1}$ Optoelectronics Research Centre, University of Southampton, Southampton SO17 1BJ, United Kingdom \\ ${ }^{2}$ Department of Chemistry and Materials Research Institute, Pennsylvania State University 16802 PA, USA \\ pm4g09@orc.soton.ac.uk
}

\begin{abstract}
Degenerate and non-degenerate two-photon absorption based modulation is demonstrated in a hydrogenated amorphous silicon core optical fiber. We show modulation using femtosecond pulses and compare this with theory.
\end{abstract}

(C) 2011 Optical Society of America

\section{Introduction}

Semiconductor core optical fibers are generating great interest as a means to integrate silicon photonics within the fiber geometry [1]. We have recently demonstrated low loss hydrogenated amorphous silicon core fibers fabricated using a high pressure chemical deposition technique [2]. Hydrogenated amorphous silicon waveguides, both on-chip and in-fiber, have been shown to have an enhanced two-photon absorption (TPA) and Kerr nonlinearity compared to their single crystal counterparts $[3,4]$. In this paper we investigate degenerate and non-degenerate TPA to demonstrate modulation of a probe signal from a pre-defined pump sequence. This cross-absorption modulation (XAM) effect is well known to exhibit ultrafast response times and transient ultrafast recovery [5], not typically observed in direct carrier injection based modulators. Centimeter lengths of silicon-core silica-clad fibers with $5.6 \mu \mathrm{m}$ core diameters are employed, with transmission losses as low as $1.5 \mathrm{~dB} / \mathrm{cm}$ at $1.54 \mu \mathrm{m}$. We demonstrate modulation extinction ratios comparable to that observed in typical silicon on-chip waveguides.

\section{Theory and Experiment}

In the propagation of strong pump pulses through silicon, TPA will occur if the sum of the energies of two incident photons bridges the band-gap energy of the material. For a narrow band pump source the process is essentially degenerate, with an absorption strength primarily dependent on the input wavelength and intensity. The process similarly works for a pump of one wavelength and a secondary probe signal at a different wavelength. This non-degenerate process induces depletion in both the strong pump and the weak probe. To demonstrate this, we experimentally employ XAM, providing theoretical fits to establish the degenerate and non-degenerate TPA strength $\beta_{\mathrm{TPA}}, \beta_{\mathrm{TPA}}^{\prime}$. For a randomly polarized input field, a set of coupled mode equations representing the influence of a pump pulse, with an intensity $I_{1}(t, z)$, on a secondary probe, with amplitude $A_{2}(t, z)$, and the material's carrier density $\mathrm{N}(\mathrm{t}, \mathrm{z})$, may be represented by [6]:

$$
\begin{gathered}
\frac{\partial I_{1}(t, z)}{\partial z}=-\alpha_{l} I_{1}(t, z)-\sigma_{\mathrm{FCA}} N(t, z) I_{1}(t, z)-\beta_{\mathrm{TPA}} I_{1}^{2}(t, z), \\
\frac{\partial A_{2}(t, z)}{\partial z}=-\frac{\alpha_{l}}{2} A_{2}(t, z)-\frac{1}{2} \sigma_{\mathrm{FCA}} N(t, z) A_{2}(t, z)-\beta_{\mathrm{TPA}}^{\prime} I_{1}(t, z) A_{2}(t, z), \\
\frac{\partial N(t, z)}{\partial t}=\frac{\beta_{\mathrm{TPA}}}{2 h v_{1}} I_{1}^{2}(t, z)-\frac{N(t, z)}{\tau} .
\end{gathered}
$$

where $\alpha_{l}$ is the linear absorption, $\sigma_{\mathrm{FCA}}$ the free carrier cross-section and $v_{1}$ the pump frequency. In the degenerate case $\beta_{\mathrm{TPA}}=\beta_{\mathrm{TPA}}^{\prime}$. We have performed pump-probe experiments in a $2 \mathrm{~cm}$ long silicon fiber to demonstrate both degenerate and non-degenerate TPA where in both cases the pump pulses had a duration of $650 \mathrm{fs}$ at $1540 \mathrm{~nm}$. For the degenerate case the probe was a weak replica of the pump, while in the non-degenerate case 1 ps probe pulses were used at $1570 \mathrm{~nm}$. Absorption of the pulses were recorded using a lock-in detection system to which it was found that both degenerate and non-degenerate modulation exhibited similar extinction ratio's as illustrated in Figs. 1 a) and b). We 

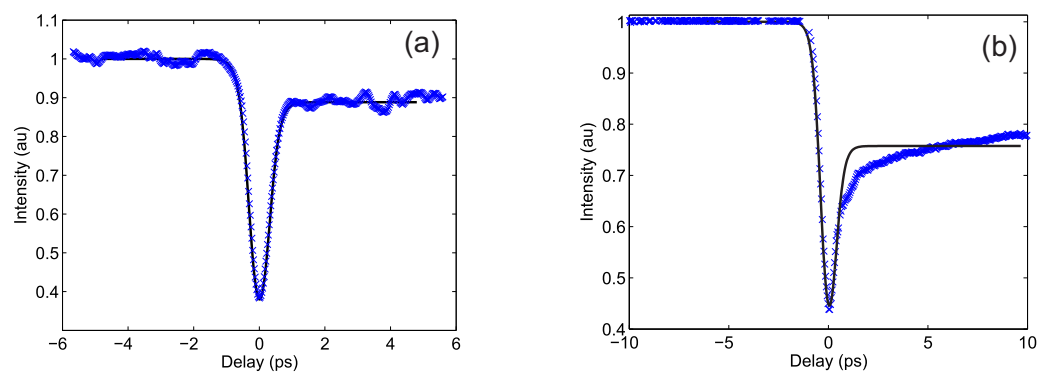

Fig. 1. Experimental measurements of (a) degenerate and (b) non-degenerate absorption. Solid lines indicate theoretical fits using Eq. (1).

investigate modulation over a range of pump powers to minimize the error in the simulated fits for the TPA strength. The estimated TPA coefficient was $\beta_{\text {TPA }} \sim 0.8 \mathrm{~cm} / \mathrm{GW}$, in agreement with that found using alternative measurement techniques [4]. The lower estimated value of the non-degenerate TPA of $\beta_{\text {TPA }}^{\prime} \sim 0.49 \mathrm{~cm} / \mathrm{GW}$ is expected based on the Kramers-Kronig relation [7]. In addition, we have also demonstrated the modulation of a CW probe at 1570nm. The result, as viewed on a bandwidth-limited oscilloscope is illustrated in Fig. 2. As expected the impulse in Fig. 2(a) shows an inverted response relative to the pump, while from Fig. 2(b) it is clear that there is a slow recovery due to carrier recombination. Using an exponential fit, the lifetime for this fiber is estimated at $86 \mathrm{~ns}$.
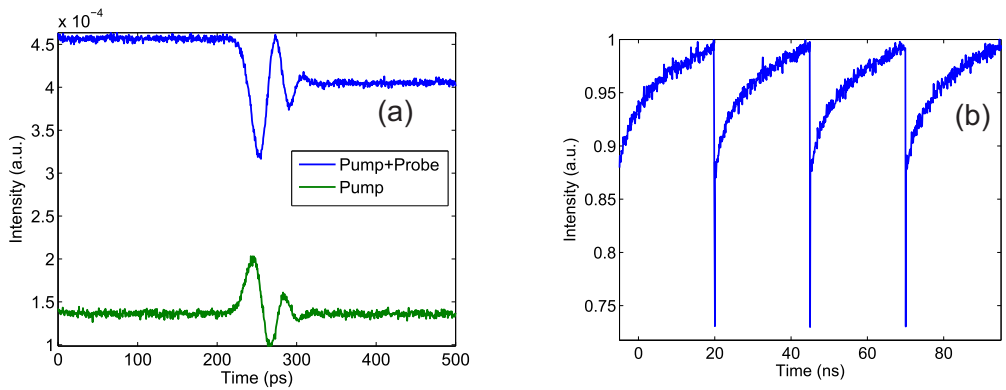

Fig. 2. Ultrafast modulation as viewed on a bandwidth limited oscilloscope. (a) Impulse response of pump and modulated probe pulse (b) Presence of carrier recombination.

\section{Conclusion}

We have characterized degenerate and non-degenerate XAM in a silicon core optical fiber, demonstrating ultrafast behavior. The results are in good agreement with the theory and reveal nonlinear parameters specific to the core material. We anticipate that these fibers should find applications in future compact in-fiber photonic devices.

\section{References}

1. D. Graham-Rowe, "Fibres get functional," Nature Photonics 5, 66-67 (2011).

2. L. Lagonigro, N. Healy, J. R. Sparks, N. F. Baril, P. J. A. Sazio, J. V. Badding, and A. C. Peacock, "Low loss silicon fibers for photonics applications," Appl. Phys. Lett. 96, 041105 (2010).

3. K. Narayanan and S. F. Preble, "Optical nonlinearities in hydrogenated amorphous silicon waveguides," Opt. Express 18, 8998-9005 (2010).

4. P. Mehta, N. Healy, N. F. Baril, P. J. A. Sazio, J. V. Badding, and A. C. Peacock, "Nonlinear transmission properties of hydrogenated amorphous silicon core optical fibers," Opt. Express 16, 16826-16831 (2010).

5. Y. Shoji, T. Ogasawara, T. Kamei, Y. Sakakibara, S. Suda, K. Kintaka, H. Kawashima, M. Okano, T. Hasama, H. Ishikawa, and M. Mori, "Ultrafast nonlinear effects in hydrogenated amorphous silicon wire waveguide," Opt. Express 18, 5668-5673 (2010).

6. J.Y. Lee, L.H. Yin, G.P. Agrawal, P.M. Fauchet, "Ultrafast optical switching based on nonlinear polarization rotation in silicon waveguides," Opt. Express 18, 11514-11523 (2010).

7. M. Sheik-Bahae, J. Wang, and E. W. Van Stryland, "Non-degenerate optical Kerr effect in semiconductors," IEEE. J. Quant. Elect 30, 249-255 (1994). 